\title{
Direct Laser Printing of Liver Cells on Porous Collagen Scaffolds
}

\author{
V. Leva*, M. Chatzipetrou*, L. Alexopoulos ${ }^{* *}$, D. S. Tzeranis ${ }^{* *}, * * *$, I. Zergioti* \\ *Department of Physics, National Technical University of Athens, Zografou 15780, Greece \\ E-mail: zergioti@central.ntua.gr \\ ** Department of Mechanical Engineering, National Technical University of Athens, Zografou 15780, Greece \\ ${ }^{* * *}$ Institute of Molecular Biology and Biotechnology, Foundation for Research and Technology-Hellas, Herakleion 70013, \\ Greece
}

\begin{abstract}
Over the past years, direct printing techniques have been increasingly utilized in biomedical research applications including the fabrication of biosensors by printing biomaterials on electronic devices [1] and the fabrication of cell constructs for tissue engineering and regenerative medicine [2,3]. In particular, the combination of natural and/or synthetic scaffolds (that provide cells with a 3D extracellular matrix analog that can support and modulate their phenotypes) with emerging direct printing techniques that can place specific cell types into specific locations, can lead to the development of novel 3D tissue models and cell constructs whose sophistication lies beyond the current state of the art.

In this work we introduce a direct laser induced forward transfer (LIFT) technique for the targeted printing of hepatocyte cancer cells (line Huh7) at precise locations on porous collagen scaffolds (PCS), in order to form patterns on the scaffold [4]. We present a detailed study of the LIFT conditions that enable repeatable printing of viable cells on the scaffold and investigate the dynamic mechanisms that occur during the cell printing process by time-resolved imaging via a high-speed camera. Cell viability and the ability to form specific cell structures inside the PCS was examined $2 \mathrm{~h}$ and $24 \mathrm{~h}$ after cell printing via fluorescence microscopy.

DOI: $10.2961 /$ jlmn.2018.03.0015
\end{abstract}

Keywords: laser printing, LIFT, direct write, cells, cell printing, porous collagen scaffolds.

\section{Introduction}

Laser induced forward transfer (LIFT) is an orifice-free direct-write method that can print viable bioinks including living cells in preset 2D or 3D patterns. LIFT has many advantages over other direct-write techniques: It is a contactless technique that offers high spatial control (10-100 $\mu \mathrm{m}$ [5]) over the printed patterns, able to print a great variety of materials in terms of viscosity [6]. Over the past years, great attention is paid to the use of laser-assisted methods for the printing of cells, due to their potential applications in tissue engineering and generative medicine.

Othon et al. firstly reported the printing of cells using LIFT technique with 95\% cell viability [7]. Another group (Koch et al.) in 2010, created well-defined viable patterns of skin cells (fibroblasts/keratocytes) and human mesenchymal stem cell and focused on the induced DNA damage and cell survival [8]. The same year, Ovsianikov et al. fabricated 3D scaffolds by means of two-photon polymerization (2PP) and printed two cells types in different regions of the scaffold by LIFT technique [9].Then, Gaebel et al. presented the first study on the preparation of a polyester urethane cardiac patch seeded with human umbilical vein endothelial cells and human mesenchymal stromal cells in well-defined patterns printed by LIFT technique [10]. The first multi-layered, fully cellularized skin equivalent was prepared by S. Michael et al. by positioning fibroblasts and keratinocytes on top of a stabilizing matrix [11]. Finally, recent studies by Keriquel et al. focused on identifying conditions for LIFT technique printing of mesenchymal stromal cells in two different distinct geometries (dish and ring) on a mice calvaria model [12].
In the LIFT process, cells to be printed are initially embedded in liquid medium whose composition depends on the application. This cell suspension will be subsequently coated on a donor substrate which will then be irradiated by a laser pulse. Laser irradiation generates a high-pressure bubble and leads to the formation of a liquid jet. This liquid jet will ultimately form a droplet at a receiver substrate, placed in close proximity to the donor substrate.

The present study utilizes the LIFT technique to print hepatocyte cancer cell line Huh7 on porous collagen-GAG scaffolds, biomaterials with established applications in regenerative medicine implants. The work focused initially on optimizing LIFT parameters in order to successfully create patterns of viable cells on the scaffold. Viable cells can then migrate inside scaffold pores and form complex 3D cell culture systems. In this study, the authors present the dynamics of the LIFT printing of cells in liquid medium and utilized a high-speed camera to record in real time the printing phenomena that occur after laser irradiation of the donor substrate. Finally, fluorescence microscopy data are presented in order to verify that Huh7 cells survived the optimized LIFT process and formed specific cell patterns into the scaffold. The ability to localize the seeding of cells into PCS opens new prospects for the development of expanding PCS applications in regenerative medicine and in generating novel in vitro tissue models based on PCS.

\section{Materials and methods}

\subsection{Laser Induced forward transfer}

The LIFT experimental setup for cell micropattern printing, shown in Fig 1, consists of a Passat Laser COMPILER 
1064/532-200I at 532nm with a Gaussian profile (1 Hz repetition rate, 6 ps pulse duration) and a high-power imaging micromachining system. As the laser beam exits the laser source, is guided via an optical setup to a donor substrate that consists of a transparent carrier (quartz), a light absorbing gold layer $(\mathrm{Au})$, and the layer of the liquid (cell suspension in medium) to be transferred. The result of the irradiation of the donor substrate from the laser pulse, is the rise of the temperature, locally, and the creation of an air bubble in the interface between the gold layer and the liquid. The air bubble expands until the atmospheric pressure, in conjunction with the surface tension forces become larger than its inner pressure, resulting in its collapse and the formation of a jet which travels towards the receiver substrate and the printing of the droplet.

The size of the droplets can be effectively controlled by the LIFT conditions such as the laser pulse fluence (calculated by dividing the average power with the irradiated area), the distance between donor-receiver substrate and the beams' size as it is controlled by a circular aperture of variable dimensions. In the present study, the laser spot size as formed by the projection of the circular aperture, to the donor substrate, was circular with a diameter of $60 \mu \mathrm{m}$, the distance between the donor and the receiver substrate was fixed at $500 \mu \mathrm{m}$ and the laser pulse fluence for the printing of the cells onto the scaffold was $530 \mathrm{~mJ} / \mathrm{cm}^{2}$. The time-resolved images of the LIFT printing process were acquired by a highspeed camera (Fastcam mini AX-Photron) able to provide up to 540,000 frames per second.

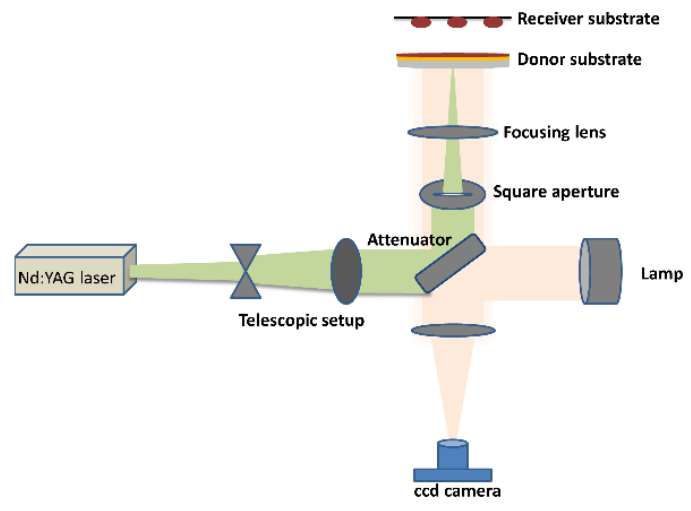

Fig. 1 Schematic representation of LIFT setup.

\subsection{Cell culture}

HuH-7 is a well-differentiated hepatocyte derived cellular carcinoma cell line, originally taken from a liver tumor in a 57-year-old Japanese male in 1982. Huh7 cells were grown on 6-well plates in RPMI 1640 medium (Biosera, France) supplemented with $10 \%$ fetal bovine serum (FBS) and $1 \%$ penicillin-streptomycin inside a humidified incubator with a temperature of $37^{\circ} \mathrm{C}$ and $5 \%$ concentration of $\mathrm{CO}_{2}$. When the confluence of the cells was $80 \%$ (usually 2 days later), the medium was removed, cells were washed in PBS and were detached from the well using $300 \mu \mathrm{l} 0.05 \%$ trypsin. After pelleting cells by centrifugation (380xg for 5 min), cells were re-suspended in fresh RPMI 1640 medium to a concentration of $5000 \mathrm{cells} / \mu \mathrm{l}$.

\subsection{Porous collagen scaffolds (PCS)}

Porous collagen-based scaffolds are biomaterials with long-established applications as grafts for regenerative medicine [13]. This study utilized porous collagen-GAG scaffolds, similar to FDA-approved grafts utilized clinically in skin regeneration [14]. Scaffolds were fabricated by freezedrying a collagen suspension ( $5 \mathrm{mg} / \mathrm{ml}$ microfibrillar collagen I, $0.44 \mathrm{mg} / \mathrm{ml}$ chondroitin-6-sulfate, dissolved in $50 \mathrm{mM}$ acetic acid) by cooling to $-40^{\circ} \mathrm{C}$ with a step of $1{ }^{\circ} \mathrm{C} / \mathrm{min}$ [15] and then cross-linked via dehydro-thermal treatment at 105 ${ }^{\circ} \mathrm{C}, 50$ mTorr for $24 \mathrm{~h}$.

\subsection{Cell staining and imaging}

Immediately after the LIFT cell printing procedure, cellseeded scaffolds were incubated in a $\mathrm{CO}_{2}$ humidified incubator with a temperature of $37^{\circ} \mathrm{C}$ and $5 \%$ concentration of $\mathrm{CO}_{2}$, for $30 \mathrm{~min}$. Samples were then stained in a live-dead staining solution ( $3 \mu \mathrm{M}$ calcein AM, $1 \mu \mathrm{M}$ propidium iodide, $3 \mu \mathrm{g} / \mathrm{ml}$ hochst 33342) for $1 \mathrm{~h}$ inside the incubator, washed in PBS and imaged in a Juli fluorescent microscope system (NanoEnTek) using fluorescence filter sets for DAPI (Hoechst dye), GFP (calcein AM dye) and RFP (propidium iodide dye) using a 4x objective lens. Images were processed in ImageJ software

\section{Results and discussion.}

\subsection{Time-resolved imaging of LIFT of cells.}

In the first part of the study, we investigate the LIFT dynamics during LIFT printing of a cell suspension. Towards this goal, a side view imaging setup with the use of the high speed camera was utilized to record the formation of the air bubble soon after laser irradiation, the traveling liquid jet, and the final deposition of the liquid droplet. The use of a high speed camera enabled the recording of each liquid jet at several time frames.

Fig. 2 depicts snapshots of liquid material ejection for laser fluence $530 \mathrm{~mJ} / \mathrm{cm}^{2}$. Results show that $5.8 \mu \mathrm{s}$ after the irradiation of the donor, a conical directional jet is created. As the jet expands, we observe directional movement of the liquid towards the receiver substrate. At $17.6 \mu$ s, the torque of the liquid jet, results to the creation of additional jets in the periphery of the main directional jet. For approximately 200 $\mu$ s there is a liquid neck between the donor and the receiver substrate which leads to a continues supply of liquid from the donor substrate to the receiver substrate. At $247 \mu$ s, the liquid neck breaks very close to the donor substrate and the remaining liquid travels in the form of a droplet towards the receiver. The final liquid droplet observed at the receiver, oscillates for almost 200 additional $\mu$ s. The whole LIFT process for each liquid droplet lasts about $400 \mu \mathrm{s}$.

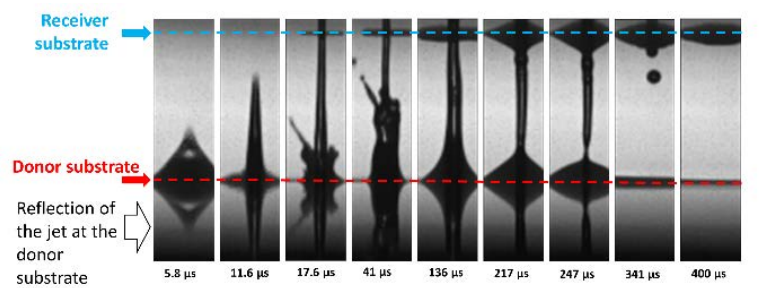

Fig. 2 LIFT of a Huh7 cell suspension on a porous collagen scaffold at fluence of $530 \mathrm{~mJ} / \mathrm{cm}^{2}$, velocity of the jet is 38.6 $\mathrm{m} / \mathrm{s}$ 
Changes on the laser pulse fluence may lead to the formation of jets with different diameter and shape. Apart from the shape of the jet, a key parameter of interest is the travel velocity of the liquid jet. Selecting lower laser pulse fluence resulted in lower values of jet travel velocity (therefore the whole printing process becomes slower) and lower liquid impact pressure. The impact pressure corresponds to the collision pressure of the liquid droplet with the receiver substrate, as the jet arrives at the receiver substrate for the first time during the impact. The impact pressure $P$ can be estimated based on the liquid jet travel velocity according to the dynamic pressure equation, (where $u$ is the jet travel velocity and $\rho$ is the liquid density).

$$
P=\frac{1}{2} p u^{2}
$$

We recorded the dynamics of the LIFT process for four different laser pulse fluences, measured the jet travel velocity and calculated the liquid impact pressure. Results are presented in Table 1.

Table 1 Measured liquid jet travel velocity and estimated resulting impact pressure for LIFT of Huh7 cell suspension, as a function of laser pulse fluence.

\begin{tabular}{|c|c|c|}
\hline $\begin{array}{c}\text { Fluence } \\
\left(\mathbf{m J} / \mathbf{c m}^{2}\right)\end{array}$ & Velocity $(\mathrm{m} / \mathrm{s})$ & $\begin{array}{c}\text { Impact pres- } \\
\text { sure (MPa) }\end{array}$ \\
\hline 330 & 8.0 & 0.03 \\
\hline 420 & 21.0 & 0.22 \\
\hline 500 & 27.9 & 0.40 \\
\hline 530 & 38.6 & 0.77 \\
\hline
\end{tabular}

By increasing the laser pulse fluence, the liquid jet travel velocity increases linearly. This is expected taking into account that higher amount of energy is given from the laser pulse, part of which transforms to jet kinetic energy. The dynamic impact pressure follows a parabolic form and it can be observed that for $530 \mathrm{~mJ} / \mathrm{cm}^{2}$, which is the optimum laser pulse fluence for the printing of cells, the estimated value of impact pressure is $0.8 \mathrm{MPa}$.

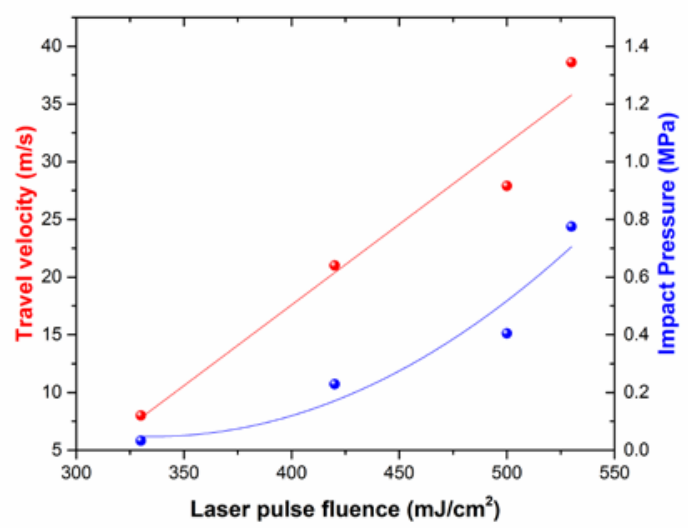

Fig. 3 The calculated front velocity as a function of the laser fluence (left axis); the corresponding impact pressure as a function of the laser fluence (right axis).

\subsection{Printing cells into PCS via LIFT}

After the successful LIFT of cells into PCS, cell-printed scaffolds were filled carefully with medium and were incubated for $30 \mathrm{~min}$. Cell patterns and cell survival was verified by fluorescence microscopy using an established live/dead assay. The viability and spatial location of cells printed onto scaffolds were detected via a fluorescence live/dead assay: The green emission of the Calcein AM dye stains the cytosol of live cells. The blue emission of cell membrane-permeable Hoechst33342 dye stains nuclei of both live and dead cells. The red emission of cell membrane-impermeable propidium iodine (PI) dye stains nuclei of dead cells. Cells were stained for 1 hour at $37^{\circ} \mathrm{C}$, washed in fresh medium and imaged in a Juli ${ }^{\mathrm{TM}}$ Stage fluorescence microscope (NanoEntek Inc., Seoul, Korea) using a 10x or a 20x objective lens. The number of printed cells was determined based on Hoechst33342 staining.

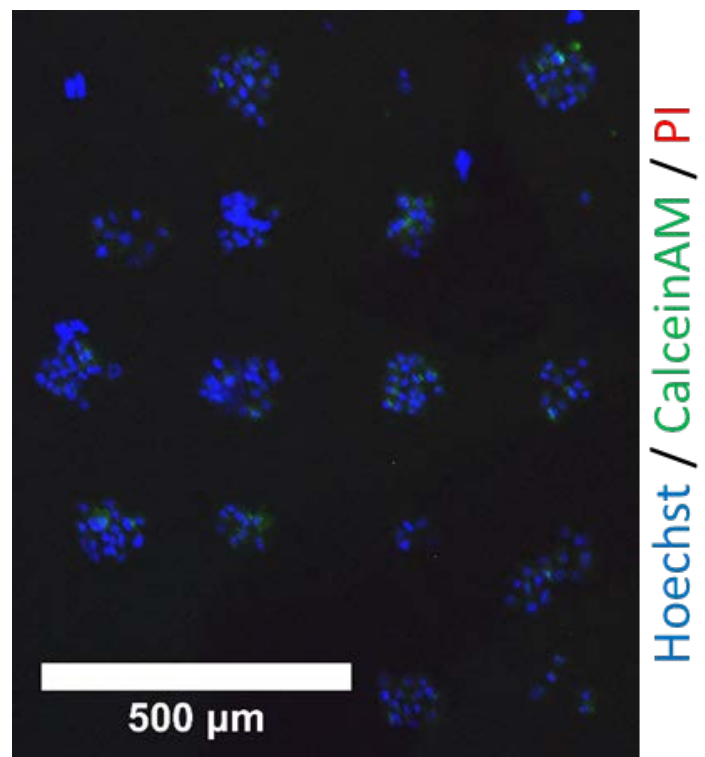

Fig. 4 Fluorescence image Huh7 microarray LIFT-printed onto a porous collagen scaffold, 2 hours after printing.

Fig. 4 shows a micro-array of cells printed onto a porous collagen scaffold via LIFT, $2 \mathrm{~h}$ after the printing process. Cells were placed in a checkboard pattern on the scaffold with high spatial control. Each liquid droplet deposited by LIFT had a diameter of $100 \mu \mathrm{m}$ while each droplet contained 16 to 20 cells. Fluorescence live/dead staining suggests that $2 \mathrm{~h}$ after printing all cells stained positively for calcein AM and no cells stained for PI, indicating cell viability of $100 \%$. 


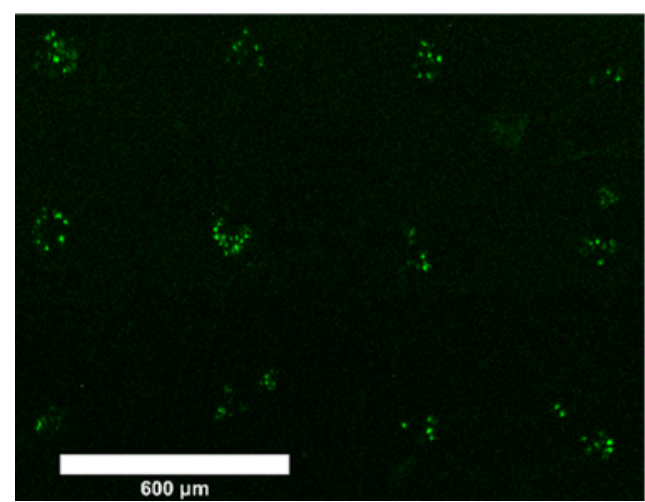

Fig. 5 Fluorescence image of a Huh7 microarray LIFT-printed onto a porous collagen scaffold, 24 hours after printing.

In order to test the viability of cells several hours after the LIFT printing process, we incubated the cell printed scaffolds for $24 \mathrm{~h}$ in cell culture medium, stained them using the live-dead stain mix and imaged them in a fluorescence an established live/dead microscope. Results show that 24h post-printing cells adhered well on the collagen (Fig. 5) and remained viable based on the presence of calcein AM dye emission and absence of PI emission. Furthermore, imaging revealed that the checkboard pattern imposed to cells during LIFT was still present.

\section{Conclusions}

In this work, we introduce the LIFT technique as a means for precise placement of cells in specific patterns on porous

\section{References}

[1] G. Tsekenis, M.K. Filippidou, M. Chatzipetrou, V. Tsouti, I. Zergioti and S. Chatzandroulis: Sensors Actuators B Chem., 208, (2015) 628.

[2] F. Guillemot, B. Guillotin, A. Fontaine, M. Ali, S. Catros, V. Kériquel, J.C. Fricain, M. Rémy, R. Bareille and J. A. Vilamitjana: MRS Bull., 36, (2011) 1015.

[3] W. Zhu, X. Ma, M. Gou, D. Mei, K. Zhang and S. Chen: Curr. Opin. Biotechnol., 40, (2016) 103.

[4] I.V. Yannas, D.S Tzeranis and B.A Harley, P.T.C So:

Philos Trans A Math Phys. Eng. Sci., 368, (2010) 2123.

[5] B. Guillotin, A. Souquet, S. Catros, M. Duocastella, B. Pippenger, S. Bellance, R. Bareille, M. Remy, L. Bordenave , J. Amedee and F. Guillemot: Biomaterials, 31, (2010) 7250.

[6] M. Makrygianni, A. Millionis, C. Kryou, I. Trantakis, D. Poulikakos and I. Zergioti: Adhesive Fluids: Adv. Mater. Interfaces, 5, (2018) 1800440.

[7] C.M Othon, X. Wu, J.J Anders and B.R Ringeisen: Biomed. Mater., 3, (2008) 034101.

[8] L. Koch, S. Kuhn, H. Sorg, M. Gruene, S. Schlie, R. Gaebel, B. Polchow, K. Reimers, S. Stoelting, N. Ma, P.M collagen scaffold. We demonstrated the technique by printing Huh7 cells onto porous collagen-GAG scaffolds and demonstrated that cells were placed at desired patterns and were viable 2 and 24 hours post-printing. We observed that 24h after printing, huh7 cells stained positively from calcein AM, adhered onto porous collagen scaffolds, and were found at the specific positions where they were initially placed by LIFT. This work demonstrates that LIFT cell printing provides a novel way of seed cells inside porous scaffolds, enabling the development of a new generation of 3D cell cultures inside porous collagen scaffold for tissue engineering applications, or novel custom-made implants for regenerative medicine applications.

\section{Acknowledgments}

(1) This research is implemented through IKY scholarships programme and co-financed by the European Union (European Social Fund - ESF) and Greek national funds through the action entitled "Reinforcement of Postdoctoral Researchers", in the framework of the Operational Programme "Human Resources Development Program, Education and Lifelong Learning” of the National Strategic Reference Framework (NSRF) 2014 - 2020.

(2) DST acknowledges financial support from the Marie Curie Actions of EU Horizon 2020 under REA grant agreement DLV-658850.

(3) This research is implemented through ESPA 2014-2020 programme through the action entitled Synergy of ELILASERLAB Europe, HIPER\&IPERION-CH.gr in the ramework of the NTUA's Participation in the project HELLAS-CH

Vogt, G. Steinhoff and B. Chichkov: Tissue Eng. Part C Methods, 16, (2010) 847.

[9] A. Ovsianikov, M. Gruene, M. Pflaum, L. Koch, F. Maiorana, M. Wilhelmi, A. Haverich and B. Chichkov: Biofabrication, 2, (2010) 014104.

[10] R. Gaebel, N. Ma, J. Liu, J. Guan, L. Koch, C. Klopsch, M. Gruene, A. Toelk, W. Wang, P. Mark, F. Wang, B. Chichkov, W. Li and G. Steinhoff: Biomaterials, 32, (2011) 9218.

[11] S. Michael, H. Sorg, C. T Peck, L. Koch, A. Dei-wick, B. Chichkov, P.M Vogt and K. Reimers: PLoS One, 8, (2013) 57741.

[12] V. Keriuel, H. Oliveira, M. Rémy, S. Ziane, S. Delmond, B. Roussea, S. Rey, S. Catros, J. Amédée, F. Guillemot and J.C Fricain: Sci. Rep., 7, (2017) 1778.

[13] I. V. Yannas, J. F. Burke, D. P. Orgill and E. M. Skrabut: Science, 215, (1982) 174.

[14] I. V. Yannas, D. S. Tzeranis, B. A. Harley and P. T. So: Philos Trans A Math Phys. Eng. Sci., 368, (2010) 2123.

[15] F. J. O'Brien, B. A. Harley, I. V. Yannas and L. Gibson: Biomaterials, 25, (2004) 1077. 